\title{
On Prognosis of Profit with Changing of Quantity of Employees of In- dustrial Enterprises
}

\author{
E.L. Pankratov ${ }^{1,2}$ \\ ${ }^{1}$ Nizhny Novgorod State University \\ Nizhny Novgorod, Russia \\ ${ }^{2}$ Nizhny Novgorod State Technical University \\ Nizhny Novgorod, Russia \\ Email: elp2004 [AT] mail.ru
}

\begin{abstract}
This paper presents a model for choosing of quantity of employees of an industrial enterprise, which necessary to obtain the required profit. This model allows predicting the possibility of reducing the losses of the enterprise associated with working in the enterprise of an excessive number of employees (wages, payment for the treatment of sick employees, training new employees, ...). An analytical technique is proposed for solving the differential equation used in the model.
\end{abstract}

Keywords --- industrial enterprise; choosing of quantity of employees; model for choosing of quantity of employees; analytical approach for analysis of model

\section{INTRODUCTION}

One of the most important factors, which determines increasing of competitiveness and the successful operation of industrial enterprises, is the development of all spheres of its activity [1,2]. In this situation enterprises must develop effective methods and adhere to the strategic concept of innovation development [3-5]. This paper presents a model for choosing of quantity of employees of an industrial enterprise, which necessary to obtain the required profit. This model allows predicting the possibility of reducing the losses of the enterprise associated with working in the enterprise of an excessive number of employees (wages, payment for the treatment of sick employees, training new employees, ...). An analytical approach was introduced to solve the differential equation used in the model.

\section{METHOD OF SOLUTION}

Let us analyze the changing of the quantity of employees of the enterprise with account the desired profit and the maximum possible value of the enterprise's personnel costs by solving the following initial problem

$$
\frac{d Y(t)}{d t}=a \cdot Y(t)+b \cdot Y(t-\tau)+c(t)+d(t)
$$

with initial condition

$$
Y(0)=0 .
$$

Here $Y(t)$ is the quantity of employees; $a$ and $b$ are the parameters of model, which should be determined by using empirical data; $c(t)$ is the required enterprise profit; $d(t)$ is the maximum possible expenses of the enterprise related to the hiring of new employees (salary, payment for the treatment of sick employees, training of new employees, $\ldots$ ); $\tau$ is the delay (i.e. $Y(t-\tau)$ is the number of employees hired or laid off over time $\tau$ ). In this article, we consider the simplest case of a single delay. Framework of the introduced approach, if necessary, a larger number of delays could be taken into account. Next, we transform equation (1) to the integral form (1a)

$$
Y(t)=a \cdot \int_{0}^{t} Y(\theta) d \theta+b \cdot \int_{0}^{t} Y(\theta-\tau) d \theta+\int_{0}^{t} c(\theta) d \theta+\int_{0}^{t} d(\theta) d \theta .
$$

We solve Eq. (1) using the Bubnov-Galerkin approach [6]. Framework this approach, we determine solution to this equation in the form of a sum of exponential functions

$$
Y(t)=d_{1} e^{-a t}+d_{2} e^{-b t} .
$$


These functions are the most natural solutions of equation (1) framework the classical theory of differential equations [7]. Substitution of function (3) into equation (1) leads to the following result

$$
d_{1} e^{-a t}+d_{2} e^{-b t}=a \cdot \int_{0}^{t}\left(d_{1} e^{-a \theta}+d_{2} e^{-b \theta}\right) d \theta+
$$

$$
+b \cdot \int_{0}^{t}\left(d_{1} e^{-a \theta} e^{a \tau}+d_{2} e^{-b \theta} e^{b \tau}\right) d \theta+\int_{0}^{t} c(\theta) d \theta+\int_{0}^{t} d(\theta) d \theta
$$

Calculation of these integrals in the right side of the equation gives a possibility to obtain the following relation

$$
d_{1} e^{-a t}+d_{2} e^{-b t}=d_{1}\left(1-e^{-a t}\right)+d_{2} \frac{a}{b}\left(1-e^{-b t}\right)+
$$

$$
+d_{1} \frac{b}{a}\left(1-e^{-a t}\right) e^{a \tau}+d_{2}\left(1-e^{-b t}\right) e^{b \tau}+\int_{0}^{t} c(\theta) d \theta+\int_{0}^{t} d(\theta) d \theta
$$

Let us multiply the left and right sides of the above relation by $e^{-a}{ }^{t}$ or $e^{-b}{ }^{t}$ and integrate from 0 to $\infty$. As a result of this operation, we obtain equations for calculating the coefficients $a$ and $b$

$$
\left\{\begin{array}{l}
d_{1} \frac{b^{2} e^{a \tau}}{a(a+b)}+d_{2} \frac{b e^{b \tau}}{a+b}=-\int_{0}^{\infty} e^{-a t} c(t) d t-\int_{0}^{\infty} e^{-a t} d(t) d t \\
d_{1} \frac{b}{a+b}\left(1-\frac{a}{b}-e^{a \tau}\right)+d_{2} \frac{1}{2}\left(1-\frac{a}{b}-e^{b \tau}\right)=\int_{0}^{\infty} e^{-b t} c(t) d t+\int_{0}^{\infty} e^{-a t} d(t) d t
\end{array}\right.
$$

The solution of this system of equations leads to the following result

$$
\left\{\begin{array}{l}
d_{1}=\frac{1}{\frac{1}{2} e^{a \tau}\left(1-a^{-1} b-e^{b \tau}\right)-\frac{e^{b \tau}}{1+a^{-1} b}\left(1-\frac{a}{b}-e^{a \tau}\right)}\left\{e^{a \tau}\left[\int_{0}^{\infty} e^{-b t} c(t) d t+\int_{0}^{\infty} e^{-b t} d(t) d t\right]+\right. \\
\left.+a^{-1} b\left(1-a b^{-1}-e^{a \tau}\right)\left[\int_{0}^{\infty} e^{-a t} c(t) d t+\int_{0}^{\infty} e^{-a t} d(t) d t\right]\right\} \\
d_{2}=-\frac{1}{\frac{b e^{a \tau}}{2 a}\left(1-\frac{a}{b}-e^{b \tau}\right)-\frac{b e^{b \tau}}{a+b}\left(1-\frac{a}{b}-e^{a \tau}\right)}\left\{e^{b \tau}\left[\int_{0}^{\infty} e^{-b t} c(t) d t+\int_{0}^{\infty} e^{-b t} d(t) d t\right]+\right. \\
\left.+\frac{1}{2}\left(\frac{a}{b}+1\right)\left(1-\frac{a}{b}-e^{b \tau}\right)\left[\int_{0}^{\infty} e^{-a t} c(t) d t+\int_{0}^{\infty} e^{-a t} d(t) d t\right]\right\}
\end{array}\right.
$$

In the simplest case at $c=c_{0}$ and $d=d_{0}$ we obtain

$$
\left\{\begin{array}{l}
d_{1}=2 \frac{c_{0}}{a} \frac{(a+b)\left[a^{2} e^{a \tau}+b\left(b-a-b e^{a \tau}\right)\right]}{e^{a \tau} b(a+b)\left(a-b-a e^{b \tau}\right)-2 a^{2} e^{b \tau}\left(b-a-b e^{a \tau}\right)} \\
d_{2}=-\frac{c_{0}}{b^{2}} \frac{(a+b)^{2}\left(b-a-b e^{b \tau}\right)+2 a e^{b \tau}}{e^{a \tau}(a+b)\left(b-a-b e^{b \tau}\right)-2 a e^{b \tau}\left(b-a-b e^{a \tau}\right)}
\end{array}\right.
$$

\section{DISCUSSION}

We use the above relations to analyze the quantity of employees taking into account changes in profit and expenses. Fig. 1 shows typical dependences of the quantity of employees on time for various values of the parameters $a$ and $b$ with constant values of profit $c_{0}$ and losses $d_{0}$. Fig. 2 shows typical dependences of the quantity of employees on the value of parameter $a$ for various values of parameter $b$, at different values of time $t$ and constant values of profit $c_{0}$ and losses $d_{0}$. Dependences of the quantity of employees on parameter $b$ are similar to dependences on Fig.2 (see Fig. 3). Dependences of the quantity of employees on the delay $t$ are also decreasing functions (see Fig. 4). Figs. 5 and 6 show dependences of quantity of employees on parameters $c_{0}$ and $d_{0}$, respectively, at different values of time $t$ and at constant values of parameters $a$ and $b$. 


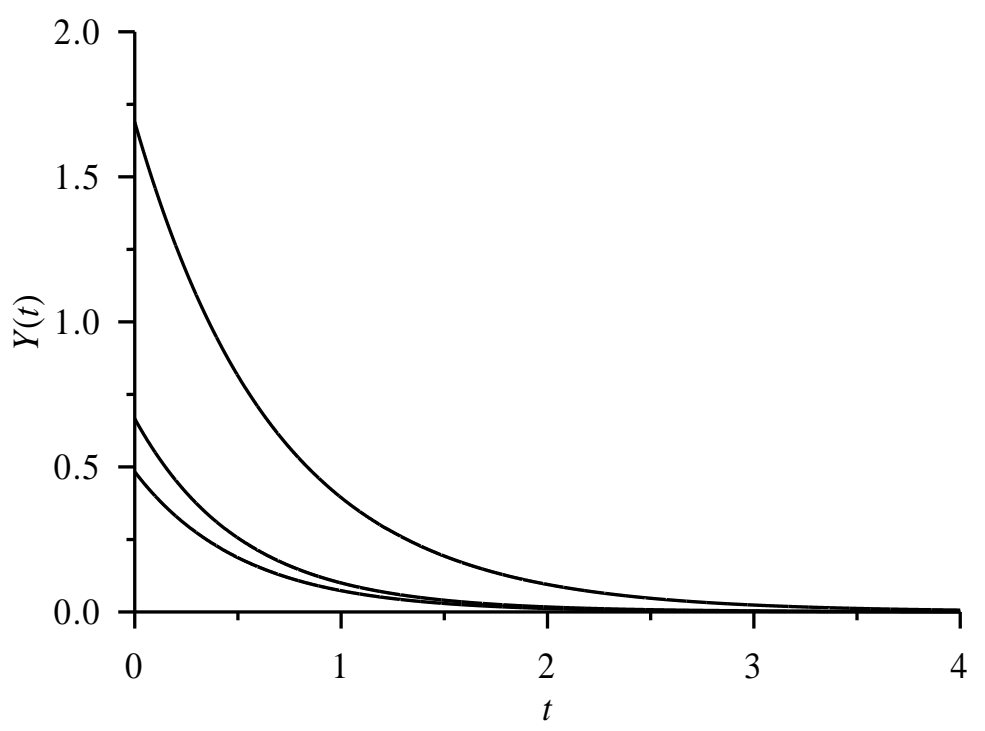

Fig. 1. Typical dependences of the quantity of employees on time for various values of the parameters $a$ and $b$ with delay $\tau$ and constant values of profit $c_{0}$ and losses $d_{0}$

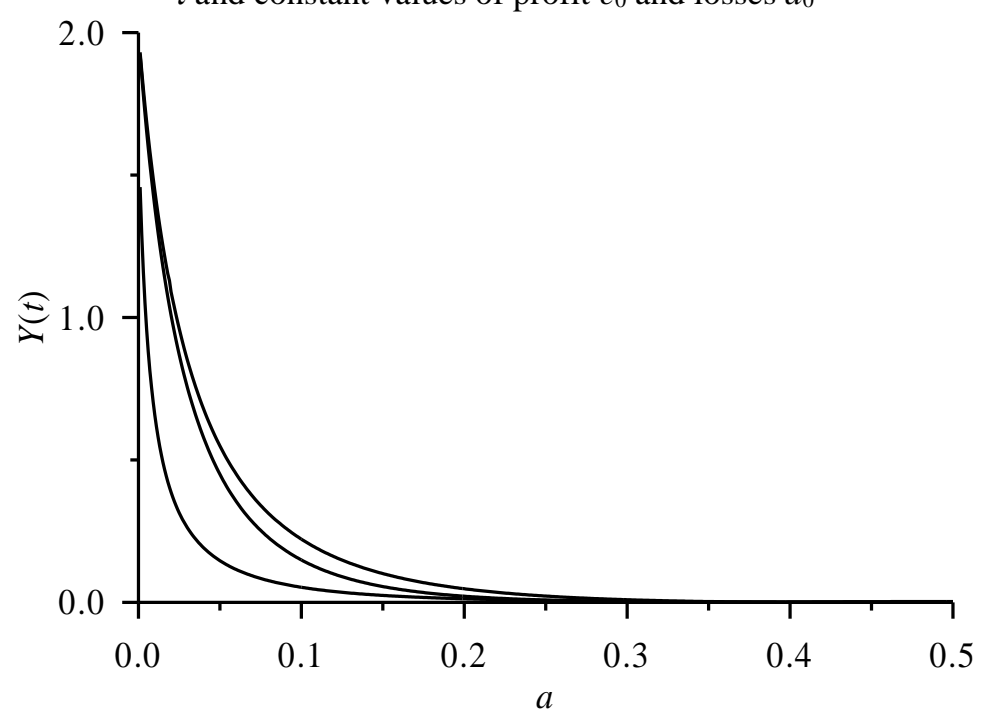

Fig. 2. Typical dependences of the quantity of employees on the value of parameter $a$ for various values of parameter $b$, at different values of time $t$ and delay $\tau$, and constant values of profit $c_{0}$ and losses $d_{0}$

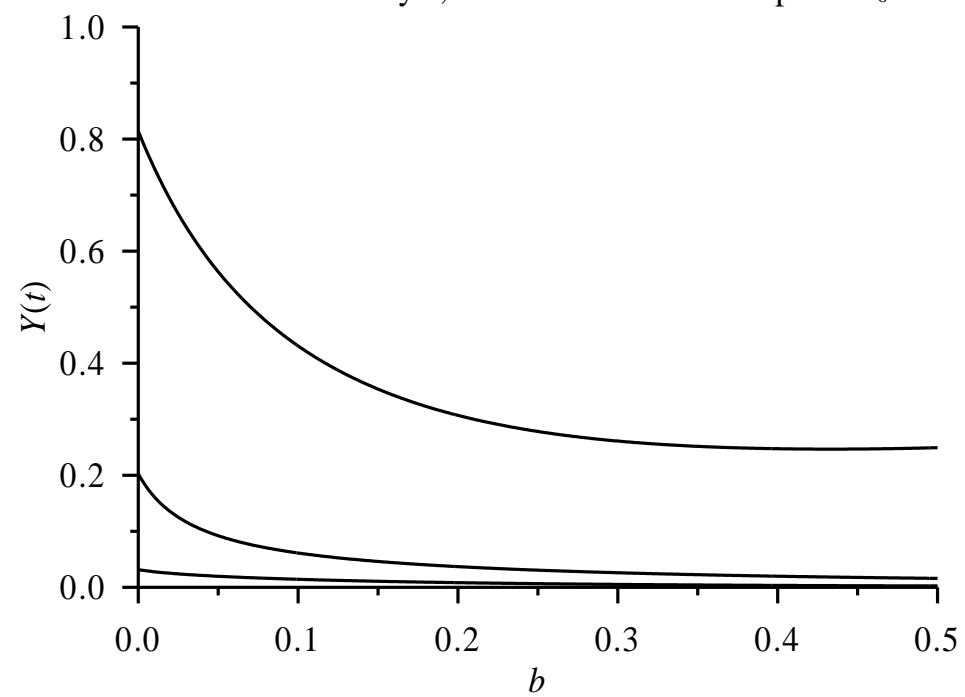

Fig. 3. Typical dependences of the quantity of employees on the value of parameter $b$ for various values of parameter $a$, at different values of time $t$ and delay $\tau$, and constant values of profit $c_{0}$ and losses $d_{0}$ 


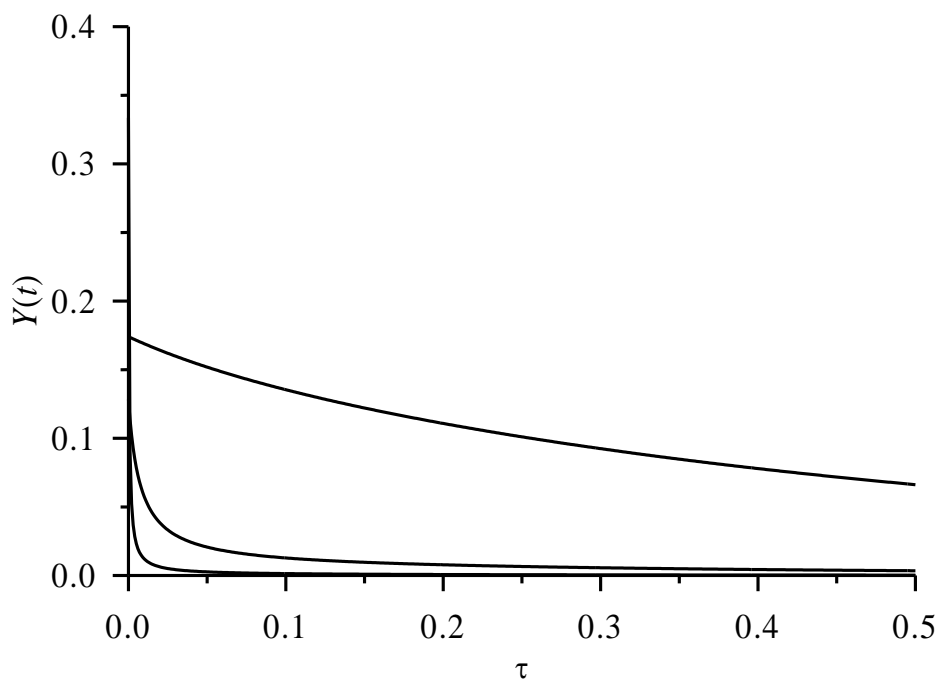

Fig. 4. Typical dependences of the quantity of employees on the value of delay $\tau$ for various values of parameters $a$ and $b$, at different values of time $t$ and constant values of profit $c_{0}$ and losses $d_{0}$

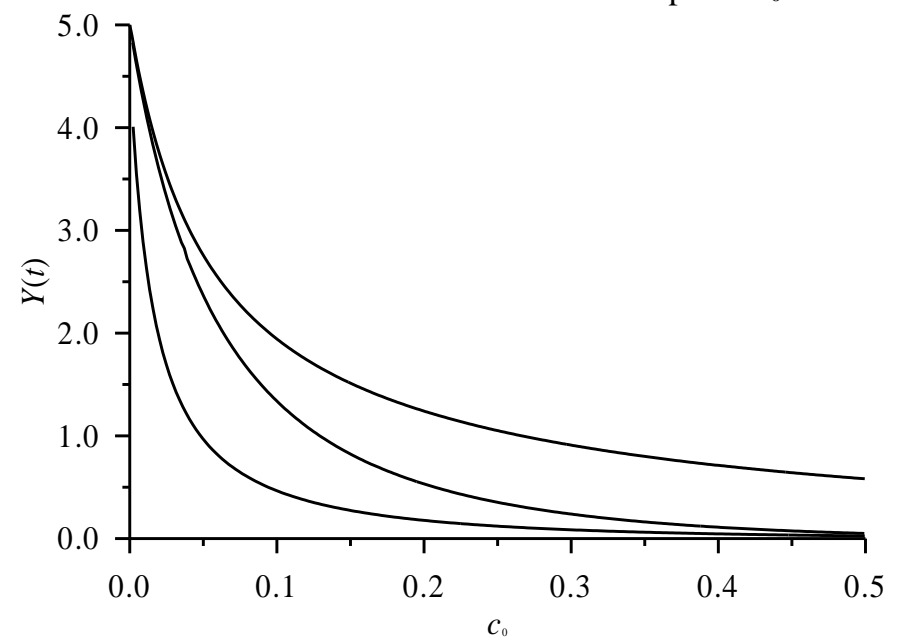

Fig. 5. Typical dependences of the quantity of employees on the value of profit $c_{0}$ for various values of parameters $a$ and $b$, at different values of time $t$ and delay $\tau$, and constant values of losses $d_{0}$

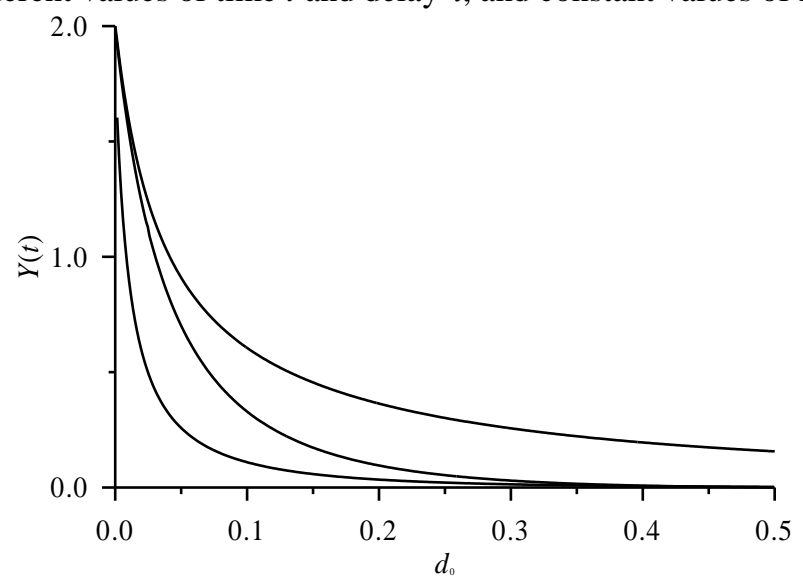

Fig. 6. Typical dependences of the quantity of employees on the value of losses $d_{0}$ for various values of parameters $a$ and $b$, at different values of time $t$ and delay $\tau$, and constant values of profit $c_{0}$

\section{CONCLUSION}

A model for predicting of quantity of employees of an industrial enterprise necessary to obtain the desired profit is introduced. The model leads to prediction of possibility of reducing the costs of the enterprise associated with the work at the enterprise of an excessive quantity of employees. The analytical approach to solve differential equation used in the model is considered. 


\section{REFERENCES}

[1] Nathaphan S, Chunhachinda P., "Estimation risk modeling in optimal portfolio selection: an empirical study from emerging markets", Economics research international, vol. 210, article ID 340181, 2010.

[2] Prat J., "Dynamic contracts and learning by doing" Mathematics and financial economics. vol. 9, no 3, pp. 169-193, 2015.

[3] Wu Ch.W., Hung W.Zh., "Real national income average growth rate: a novel economic growth and social fair evaluation index", Economics research international, vol. 2010, article ID 678927, 2010.

[4] Villeneuve St., Warin X., "Optimal Liquidity management and Hedging in the presence of a non-predictable investment opportunity", Mathematics and financial economics, vol. 8, no 2, pp. 193-227, 2014.

[5] Mertzanis Ch., "Short selling regulation, return volatility and market volatility in the Athens Exchange", Studies in economics and finance, vol. 34, no 1, pp. 82-104, 2017.

[6] Krasnov M.L., Kiselev A.I., Makarenko G.I., Integral equations, Science, Soviet Union, 1976.

[7] Korn G., Korn T., Mathematical Handbook for scientists and engineers. Definitions, theorems and formulas for reference and review. Second edition, McGraw-Hill Book Company, USA, 1968. 